\begin{tabular}{|c|l|}
\hline Title & Physical and functional interactions between Daxx and TSG101. \\
\hline Author(s) & Muromoto, Ryuta; Sugiyama, Kenji; Y amamoto, Tetsuy a; Oritani, Kenji; Shimoda, Kazuy a; Matsuda, Tadashi \\
\hline Citation & $\begin{array}{l}\text { Biochemical and Biophysical Research Communications, 316(3), 827-833 } \\
\text { https://doi.org/40.1016/.bbrc.2004.02.126 }\end{array}$ \\
\hline Issue Date & 20040409 \\
\hline Doc URL & http://hdl.handle.net/2115/28120 \\
\hline Type & article (author version) \\
\hline File Information & BBRC316-3.pdf \\
\hline
\end{tabular}

Instructions for use 


\section{Title: Physical and Functional Interactions between Daxx and TSG101}

Running title: Interactions between Daxx and TSG101

Authors: Ryuta Muromoto*, Kenji Sugiyama\#, Tetsuya Yamamoto*, Kenji Oritani $\square$, Kazuya Shimoda** and Tadashi Matsuda*

Affiliation: *Department of Immunology, Graduate School of Pharmaceutical Sciences, Hokkaido University, Kita-Ku Kita 12 Nishi 6, Sapporo 060-0812, Japan

\#Nippon Boehringer Ingelheim Co., Ltd., Kawanishi Pharma Research Institute, 3-10-1

Yato, Kawanishi, Hyogo 666-0193, Japan

$\square$ Department of Internal Medicine and Molecular Science, Graduate School of Medicine, Osaka University, 2-2 Yamada-oka, Suita, Osaka 565-0871, Japan

**First Department of Internal Medicine, Faculty of Medicine, Kyushu University, 3-1-1 Maidashi, Higashi-Ku, Fukuoka, Japan

Footnotes: This work was supported by Grants-in-Aid for Scientific Research from the Ministry of Education, Science, Sports and Culture in Japan, the Suhara Memorial Foundation and Uehara Memorial Foundation.

Address for manuscript correspondence: Dr. Tadashi Matsuda, Department of Immunology, Graduate School of Pharmaceutical Sciences, Hokkaido University, Kita-Ku Kita 12 Nishi 6, Sapporo 060-0812, Japan TEL: 81-11-706-3243, FAX: 81-11-706-4990, E-mail: tmatsuda@pharm.hokudai.ac.jp

Key words: Daxx, TSG101, transcription, repression 


\begin{abstract}
Daxx has been reported to mediate the Fas/JNK-dependent signals in the cytoplasm. However, several evidences have suggested that Daxx is located mainly in the nucleus and functions as a transcriptional regulator. Recently, we identified DMAP1, a TSG101-interacting protein as a Daxx binding partner by yeast two-hybrid screening. TSG101 has been shown to act as transcriptional co-repressor of nuclear hormone receptors. Here we examined whether TSG101also interacts with Daxx directly. The association of Daxx and TSG101 was confirmed using co-expressed tagged proteins. The interaction regions in both proteins were also mapped, and the cellular localization of the interaction was examined. TSG101 formed a complex with Daxx through its coiled-coil domain and co-localized in the nucleus. Furthermore, TSG101 enhanced Daxx-mediated repression of glucocorticoid receptor transcriptional activity. These results provide the novel molecular interactions between Daxx and TSG101, which establish an efficient repressive transcription complex in the nucleus.
\end{abstract}




\section{INTRODUCTION}

Daxx was first identified as a Fas-binding protein by yeast two-hybrid screening and known as a pro-apoptotic protein that can enhance Fas-mediated apoptosis through Jun N-terminal kinase (JNK) activation (1). While the interaction between Daxx and Fas indicated the importance of Daxx in cytoplasm, nuclear localization of Daxx was observed in various cell lines and the interactions of Daxx with several nuclear proteins such as the centromeric protein CENP-C, DNA methyltransferase 1 (DNMT1), Pax-3, Pax-5, ETS1, Ubc9, SUMO-1 and PML were reported (2-9). These studies suggest that Daxx may play a role in the nucleus and it has been reported that it functions as a transcriptional co-regulator by interaction with various transcription factors.

Daxx has been shown to possess transcriptional repression activity by inhibiting several transcription factors such as Pax3, ETS1 and GR through direct protein-protein interactions $(4,6,10)$. Daxx is also shown to act as a transcriptional co-activator or co-repressor of Pax5 in different cell types (5). Although the exact mechanism accounting for these observations is still unclear, the recruitment of nuclear factors possessing either histone acetyltransferase or histone deacetylase (HDAC) activity by Daxx to modulate Pax5 transcriptional activity was proposed (5). In addition, the transcriptional repression effect of Daxx could be modulated by subnuclear or subcellular compartmentalization through protein-protein interactions $(11,12,13)$. Furthermore, PML has been shown to relieve the transrepression effect of Daxx on Pax3 or GR transcriptional activity through sequestering Daxx into the PML oncogenic domains (PODs) $(10,14)$.

TSG101 was discovered as a multifunctional domain protein in a screen for tumor-susceptibility genes in NIH 3T3 fibroblasts (15). The TSG101 protein plays an 
essential role during development, as demonstrated by the early lethality observed in null mutant mouse embryos (16). Sequence analysis indicates that the TSG101 protein contains an amino-terminal region of homology to ubiquitin-conjugating enzymes (UBC E2), a proline-rich sequence with the features of a transcription transactivation domain, a leucine zipper, and a central coiled-coil domain $(16,17,18)$. It has been shown that TSG101 and the yeast homolog Vps23 have an important role in ubiquitin-mediated endosomal sorting pathways $(19,20)$. Subsequently, TSG101 has been shown to act as a transcriptional co-repressor involved in the silencing of nuclear hormone receptor induced genes through the coiled-coil domain $(21,22,23)$.

Recently, we identified DMAP1 as a novel Daxx partner using yeast two-hybrid screening (24). DMAP1 has been shown to interact directly with DNMT1 and a co-repressor, TSG101, and can mediate transcriptional repression (25).

In this study, we have examined both biochemical and functional interactions between Daxx and TSG101. Daxx and TSG101 formed a complex and co-localized in the nucleus through the coiled coil domain of TSG101. TSG101 enhanced Daxx-mediated repression of glucocorticoid receptor transcriptional activity. These results provide an important linkage between Daxx and TSG101, which establishes an efficient complex with other repressors. 


\section{MATERIALS AND METHODS}

\section{Reagents and antibodies}

Dexamethasone (DEX) was purchased from Wako Chemicals (Osaka, Japan).

Expression vectors, FLAG-tagged Daxx (26), MMTV-LUC (27), Myc-tagged TSG101 (28), were kindly provided by Dr. H. Ariga and Dr. T. Taira (Hokkaido Univ., Sapporo, Japan), Dr. S. N. Cohen (Stanford Univ. Sch. Med., Stanford, CA), and Dr. G. L. Hager (NCI, NIH, Bethesda, Maryland), respectively. Myc-tagged Daxx and TSG101 mutants (TSG101N, TSG101C, TSG101CC) were generated by polymerase chain reaction (PCR) methods and sequenced (primer sequences are available upon request). Anti-Myc and anti-Daxx antibodies were purchased from Santa Cruz Biotechnology (Santa Cruz, CA). Anti-FLAG M2 antibody was purchased from Sigma (St Louis, MO).

\section{Cell culture, transfection and luciferase assays}

Human embryonic kidney carcinoma cell line, 293T, was maintained in DMEM containing 10\% FCS and transfected by the standard calcium precipitation protocol (29). The cells were harvested $48 \mathrm{~h}$ after transfection and lysed in $100 \mu \mathrm{l}$ of PicaGene Reporter Lysis Buffer (Toyo Ink, Tokyo, Japan) and assayed for luciferase and $\beta$-galactosidase activities according to the manufacturer's instructions. Luciferase activities were normalized to the $\beta$-galactosidase activities. Three or more independent experiments were carried out for each assay.

\section{Immunoprecipitation and immunoblotting}


The immunoprecipitation and Western blotting assays were performed as described previously (29). Cells were harvested and lysed in lysis buffer (50 mM Tris-HCl, pH 7.4, $0.15 \mathrm{M} \mathrm{NaCl}$, containing $1 \% \mathrm{NP}-40,1 \mu \mathrm{M}$ sodium orthovanadate, $1 \mu \mathrm{M}$ phenylmethylsulfonyl fluoride and $10 \mu \mathrm{g} / \mathrm{ml}$ each of aprotinin, pepstatin and leupeptin). The immunoprecipitates from cell lysates were resolved on SDS-PAGE and transferred to Immobilon filter (Millipore; Bedford, MA). The filters were then immunoblotted with each antibody. Immunoreactive proteins were visualized using an enhanced chemiluminescence detection system (Amersham Pharmacia Biotech).

\section{Indirect Immunofluorescence}

Monkey COS7 cells were maintained in DMEM containing 10\% FCS transfected with Daxx and/or TSG101 Full and/or TSG101CC by the calcium phosphate precipitation protocol. $48 \mathrm{~h}$ after transfection, cells were fixed with a solution containing $4 \%$ paraformaldehyde and reacted with an anti-Myc antibody or an anti-Daxx antibody. The cells were then reacted with a FITC-conjugated anti-rabbit IgG or rhodamine-conjugated anti-mouse IgG (CHEMICON, Temecula, CA) and observed under a confocal laser fluorescent microscope. At the same time, the nuclei in the cells were stained with 4', 6-diamidino-2-phenylindole (DAPI). Images were obtained by using a Zeiss LSM 510 laser scanning microscope with an Apochromat x63/1.4 oil immersion objective and x4 zoom. 


\section{RESULTS}

\section{Association of Daxx with DMAP1 and TSG101 in 293T cells}

In the previous study, a DNMT1 associated protein, DMAP1 was shown to interact with TSG101 by yeast two-hybrid screening (25). The co-immunoprecipitation experiments also demonstrated that TSG101 interacts with DMAP1 and DNMT1 in COS7 cells (25). To confirm the association of DMAP1 with TSG101 in 293T cells, 293T cells were transfected with FLAG -tagged DMAP1 together with or without Myc-tagged TSG101. As shown in Fig. 1A, Western blot analysis of the immunoprecipitates with an anti-FLAG antibody revealed that DMAP1 interacts with TSG101 in 293T cells. Our recent study revealed that DMAP1 interacted with Daxx in cells (24). We then examine whether TSG101 also associates with Daxx in 293T cells. To examine the association of Daxx with TSG101 in 293T cells, 293T cells were transfected with FLAG -tagged Daxx together with or without Myc-tagged TSG101 and/or DMAP1. As shown in Fig. 1B, Western blot analysis of the immunoprecipitates with an anti-FLAG antibody revealed that Daxx interacts with TSG101 in 293T cells. Furthermore, an additional expression of DMAP1 with TSG101 did not enhance the association between Daxx and TSG101, suggesting that Daxx directly interacts with TSG101 in 293T cells.

To delineate which regions of Daxx is involved in Daxx/TSG101 interaction, various deletion constructs of Daxx were engineered (Fig. 2A) and subjected to analyse in co-immunoprecipitation assays in $293 \mathrm{~T}$ cells. As shown in Fig. 2A, the N-terminal deletion mutant of Daxx, Daxx(493-740) failed to interact with TSG101. In contrast, the C-terminal deletion mutant $\operatorname{Daxx(1-240)}$ or $\operatorname{Daxx}(241-492)$ was capable of interacting with TSG101. These results implicated that the N-terminal region of Daxx is necessary 
and sufficient for its interaction with TSG101. We then examined the interacting domains on TSG101 with Daxx. To delineate the domains in the TSG101 that mediate the protein-protein interaction with Daxx, co-immunoprecipitation experiments were performed with a series of mutant TSG101 proteins (Fig. 2B). Expression vectors encoding FLAG-tagged Daxx and a series of Myc-tagged TSG101 mutants were transiently transfected into 293T cells. Cells were lysed, and subjected to immunoprecipitation with an anti-FLAG antibody. Immunoprecipitates were then used in Western blot analysis with an anti-Myc antibody. As shown in Fig. 2B, the C-terminal domain of TSG101 (TSG101C; amino acid residues 220-380) interacted with Daxx. Furthermore, only coiled-coil region of the C-terminal domain of TSG101 (TSG101CC; amino acid residues 231-302) mediated the protein-protein interaction between TSG101 and Daxx. These results indicate that the N-ternimal domain of Daxx interacts with the coiled-coil domain of TSG101.

\section{Co-localization of Daxx and TSG101 in the nucleus}

To determine whether TSG101 co-localizes with Daxx in the nucleus, expression vectors for Myc-tagged TSG101 and FLAG-tagged Daxx were transfected into COS7 cells. Forty-eight hours after transfection, the cells were stained with anti-Myc and anti-Daxx antibodies, and they were visualized with rhodamine and FITC-conjugated secondary antibodies, respectively, under a confocal laser microscope. As shown in Fig. 3A, TSG101 was localized throughout the cytoplasm and nucleus, while Daxx was located mainly in the nucleus. However, they were found to be co-localized after demonstration of the merged figure, in which the red and green colors turned yellow (Fig. 3A), suggesting that both Daxx and TSG101 co-localize in the nucleus. We also examined whether the coiled-coil domain of TSG101(TSG101CC) co-localizes with 
Daxx, expression vectors for Myc-tagged TSG101CC and FLAG-tagged Daxx were transfected into COS7 cells. Interestingly, TSG101CC was predominantly localized in the nucleus and co-localized with Daxx. These results indicates that Daxx and TSG101co-localize in the nucleus through the coiled-coil domain of TSG101.

\section{TSG101 and Daxx cooperatively repress glucocorticoid receptor-mediated transcriptional activity}

Daxx has been reported to function as a transcriptional modulator in the nucleus $(4,5$, 6, 9, 30, 31). It has been also demonstrated that overexpression of Daxx suppresses glucocorticoid receptor (GR)-mediated activation of the mouse mammary tumor virus (MMTV) promoter in a human embryonic kidney carcinoma cell line, 293T cells (10). TSG101 is also shown to function as a transcriptional repressor, decreasing GR-dependent transcriptional activation by binding and repressing AF-1 domain of GR (22). To examine the functional relevance of the Daxx/TSG101 interaction in the context of GR signaling pathway, we performed the transient transfection assay using 293T cells. The GR-mediated transcriptional responses were measured by MMTV-LUC, which is one of the standard reporters for assessing GR activity (27). When 293T cells were transfected with MMTV-LUC together with an expression vector for GR and treated with dexamethasone (Dex), LUC expression was increased by about 100-fold (Fig.4A, B). As shown in Fig. 4A, overexpression of Daxx suppressed GR-mediated transactivation in a dose-dependent manner. We also examined the effect of overexpression of TSG101 on GR-mediated transactivation in 293T cells. Overexpression of TSG101 showed a moderate suppression in GR-induced MMTV-LUC activity (Fig. 4A). Interesting, in the presence of Daxx, overexpression of TSG101 more effectively suppressed GR-induced MMTV-LUC activity, although the effect was additive. 
The previous study demonstrated that the suppression of GR activity was mediated through the coiled-coil region of TSG101 (21). We then tested whether the coiled-coil domain of TSG101 mediates the suppression of GR activity together with Daxx. As shown in Fig. 4B, the Daxx-mediated suppression of GR activity was enhanced by the coiled-coil domain of TSG101. These results suggest that Daxx and TSG101 can independently act as a co-repressor for GR-mediated transcription.

\section{DISCUSSION}

Transcriptional repressor complexes often contain several proteins that can exert an efficient modulation of transcription. In our previous study, the two-hybrid screening has led us identify a TSG101 associated protein, DMAP1 as a Daxx-interacting protein (24). Furthermore, we demonstrated that Daxx and DMAP1 form a complex and additively repress GR-mediated transcription, although DMAP1 had no effect on Fas/Daxx-mediated apoptosis (24). We here show that TSG101 also interacts with Daxx directly and perform an efficient transcriptional repression with Daxx.

Daxx was originally cloned as a Fas-associated protein and binds specifically to the death domain of Fas, although Daxx by itself lacks a death domain (1). There are two independent signaling pathways downstream of Fas, involving the adapter protein Fas-associated death domain (FADD) and Daxx (32). The activation of FADD induces a protease cascade (33), while that of Daxx enhances JNK activation, leading to apoptosis (1, 33). Overexpression of Daxx enhances Fas-induced apoptosis (1, 26, 31), and the targeted disruption of the Daxx gene in mice results in embryonic lethality (2). Daxx is also involved in coupling of the type II TGF- $\beta$ receptor signaling with components of the apoptotic machinery (34). Recent studies also demonstrated that Daxx might function as 
a transcriptional co-regulator for several transcription factors such as Pax3, ETS1 and GR through direct protein-protein interactions $(4,6,10)$. Daxx recruits nuclear factors possessing either histone acetyltransferase or HDAC activity to modulate transcriptional activity $(5,9,35)$. In fact, Daxx is shown to bind to $\operatorname{HDAC2}(9,35)$. In addition, the transcriptional repression effect of Daxx could be modulated by subnuclear compartmentalization through protein-protein interactions. For example, PML has been shown to relieve the transrepression effect of Daxx through sequestering Daxx into the PODs $(9,10,14)$.

TSG101 was originally identified in a screening for genes that promote neoplastic transformation when it was inactivated (15). Recently, TSG101 has been also shown to function as a transcriptional repressor protein, decreasing GR-dependent transcriptional activation by binding and repressing AF-1 of GR through the coiled-coil domain of TSG101(22). TSG101 was also demonstrated to suppress the transcriptional activity of various nuclear receptors including ER, AR, VDR and RAR (21, 23). These studies suggest that TSG101 may function by recruiting a HDAC to GR and repressing its transcriptional activity through changes in chromatin structure. However, no data whether it is mediated by HDACs is presented yet.

The present paper describes both physical and functional interactions between Daxx and TSG101. Daxx and DMAP1, TSG101 formed a complex and co-localized in the nucleus. TSG101 enhanced Daxx-mediated repression of a GR transcriptional activity. These results provide an important linkage between Daxx and TSG101, which forms an efficient transcription repression complex in the nucleus. 


\section{ACKNOWLEDGEMENTS}

We thank Dr. H. Ariga, Dr. T. Taira, Dr. S. N. Cohen, and Dr. G. L. Hager, for their kind gifts of reagents. We also thank Dr. J. Akiyama for encouraging our work. 


\section{REFERENCES}

[1] Yang, X., Khosravi-Far, R., Chang, H. Y., and Baltimore, D. (1997) Cell 89, 1067-1076. Daxx, a novel Fas-binding protein that activates JNK and apoptosis.

[2] Michaelson, J. S., Bader, D., Kuo, F., Kozak, C., and Leder, P. (1999) Genes Dev. 13, 1918-1923. Loss of Daxx, a promiscuously interacting protein, results in extensive apoptosis in early mouse development.

[3] Pluta, A. F., Earnshaw, W. C., and Goldberg, I. G. (1998) J. Cell Sci. 111,2029-2041. Interphase-specific association of intrinsic centromere protein CENP-C with HDaxx, a death domain-binding protein implicated in Fas-mediated cell death.

[4] Hollenbach, A. D., Sublett, J. E., McPherson, C. J., and Grosveld, G. (1999) EMBO J. 18,3702-3711. The Pax3-FKHR oncoprotein is unresponsive to the Pax3-associated repressor hDaxx.

[5] Emelyanov, A. V., Kovac, C. R., Sepulveda, M. A., and Birshtein. B.K. (2002) J. Biol. Chem. 277,11156-11164. The interaction of Pax5 (BSAP) with Daxx can result in transcriptional activation in B cells.

[6] Li, R., Pei, H., Watson, D.K., and Papas, T.S. (2000) Oncogene 19,745-753. EAP1/Daxx interacts with ETS1 and represses transcriptional activation of ETS1 target genes. 
[7] Ryu, S.W., Chae, S.K., and Kim, E. (2000) Biochem. Biophys. Res. Commun.

279,6-10. Interaction of Daxx, a Fas binding protein, with sentrin and Ubc9.

[8] Ishov, A. M., Sotnikov, A. G., Negorev, D., Vladimirova, O. V., Neff, N., Kamitani, T., Yeh, E. T., Strauss, J. F., III, and Maul, G. G. (1999) J. Cell Biol. 147,221-234. PML is critical for ND10 formation and recruits the PML-interacting protein daxx to this nuclear structure when modified by SUMO-1.

[9] Li, H., Leo, C., Zhu, J., Wu, X., O'Neil, J., Park, E.J., and Chen. J.D. (2000) Mol. Cell. Biol. 20,1784-1796. Sequestration and inhibition of Daxx-mediated transcriptional repression by PML.

[10] Lin, D.Y., Lai, M.Z., Ann, D.K., and Shih, H.M. (2003) J. Biol. Chem. 278,15958-15965. Promyelocytic leukemia protein (PML) functions as a glucocorticoid receptor co-activator by sequestering Daxx to the PML oncogenic domains (PODs) to enhance its transactivation potential.

[11] Lin, D.Y. and Shih, H.M. (2002) J. Biol. Chem. 277,25446-25456. Essential role of the 58-kDa microspherule protein in the modulation of Daxx-dependent transcriptional repression as revealed by nucleolar sequestration.

[12] Ko, Y.G., Kang, Y.S., Park, H., Seol, W., Kim, J., Kim, T., Park, H.S., Choi, E.J., and Kim, S. (2001) J. Biol. Chem. 276,39103-39106. Apoptosis signal-regulating kinase 1 controls the proapoptotic function of death-associated protein (Daxx) in the cytoplasm. 
[13] Ecsedy, J.A., Michaelson, J.S., and Leder, P. (2003) Mol. Cell. Biol. 23,950-960. Homeodomain-interacting protein kinase 1 modulates Daxx localization, phosphorylation, and transcriptional activity.

[14] Lehembre, F., Muller, S., Pandolfi, P.P., and Dejean, A. (2001) Oncogene 20,1-9. Regulation of Pax3 transcriptional activity by SUMO-1-modified PML.

[15] Li, L. and Cohen, S.N. (1996) Cell 85, 319-329. Tsg101: a novel tumor susceptibility gene isolated by controlled homozygous functional knockout of allelic loci in mammalian cells.

[16] Ruland, J., Sirard, C., Elia, A., MacPherson, D., Wakeham, A., Li, L., de la Pompa, J.L., Cohen, S.N. and Mak, T.W. (2001) Proc. Natl. Acad. Sci. U S A. 98, 1859-1864. p53 accumulation, defective cell proliferation, and early embryonic lethality in mice lacking tsg101.

[17] Koonin, E.V. and Abagyan, R.A. (1997) Nat. Genet. 16, 330-331. TSG101 may be the prototype of a class of dominant negative ubiquitin regulators.

[18] Ponting, C.P., Cai, Y.D. and Bork, P. (1997) J. Mol. Med. 75, 467-469. The breast cancer gene product TSG101: a regulator of ubiquitination?

[19] Katzmann, D. J., Babst, M. and Emr, S. D. (2001) Cell 106,145-155.

Ubiquitin-dependent sorting into the multivesicular body pathway requires the function 
of a conserved endosomal protein sorting complex, ESCRT-I.

[20] Bishop, N. and Woodman, P. (2001) J. Biol. Chem. 276,11735-11742.

TSG101/mammalian VPS23 and mammalian VPS28 interact directly and are recruited to VPS4-induced endosomes.

[21] Watanabe, M., Yanagi, Y., Masuhiro, Y., Yano, T., Yoshikawa, H., Yanagisawa, J., and Kato, S. (1998) Biochem Biophys Res Commun. 245, 900-905. A putative tumor suppressor, TSG101, acts as a transcriptional suppressor through its coiled-coil domain.

[22] Hittelman, A. B., Burakov, D., Iniguez-Lluhi, J. A., Freedman, L. P., and Garabedian, M. J. (1999) EMBO J. 18,5380-5388. Differential regulation of glucocorticoid receptor transcriptional activation via AF-1-associated proteins.

[23] Sun, Z., Pan, J., Hope, W.X., Cohen, S.N. and Balk, S.P. (1999) Cancer 86,689-696. Tumor susceptibility gene 101 protein represses androgen receptor transactivation and interacts with p300.

[24] Muromoto, R., Sugiyama, K., Takachi, A., Imoto, S., Sato, N., Yamamoto, T., Oritani, K., Shimoda, K. and Matsuda, T. (2004) J. Immunol. Physical and Functional Interactions between Daxx and DNA Methyltransferase 1 Associated Protein, DMAP1 in press.

[25] Rountree, M.R., Bachman, K.E., and Baylin, S.B. (2000) Nat. Genet. 25,269-277. DNMT1 binds HDAC2 and a new co-repressor, DMAP1, to form a complex at 
replication foci.

[26] Muromoto, R., Yamamoto, T., Yumioka, T., Sekine, Y., Sugiyama, K., Shimoda, K., Oritani, K. and Matsuda, T. (2003) FEBS Lett. 540,:223-228. Daxx enhances Fas-mediated apoptosis in a murine pro-B cell line, BAF3.

[27] Bresnick, E. H., John, S., Berard, D. S., LeFebvre, P., and Hager, G. L. (1990) Proc. Natl. Acad. Sci. U S A. 87,3977-3981. Glucocorticoid receptor-dependent disruption of a specific nucleosome on the mouse mammary tumor virus promoter is prevented by sodium butyrate.

[28] Li, L., Liao, J., Ruland, J., Mak, T.W., and Cohen, S.N. (2001) Proc. Natl. Acad. Sci. U S A. 98,1619-1624. A TSG101/MDM2 regulatory loop modulates MDM2 degradation and MDM2/p53 feedback control.

[29] Matsuda, T., Yamamoto, T., Kishi, H., Yoshimura, A., and Muraguchi, A. (2000) FEBS Lett. 472,235-240. SOCS-1 can suppress CD3zeta- and Syk-mediated NF-AT activation in a non-lymphoid cell line.

[30] Michaelson, J.S. and Leder, P. (2003) J. Cell Sci. 116,345-352. RNAi reveals anti-apoptotic and transcriptionally repressive activities of DAXX.

[31] Torii, S., Egan, D. A., Evans, R. A., and Reed, J. C. (1999) EMBO J. 18,6037-6049. Human Daxx regulates Fas-induced apoptosis from nuclear PML oncogenic domains (PODs). 
[32] Chang, H. Y., Yang, X., and Baltimore, D. (1999). Proc. Natl. Acad. Sci. USA 96,1252-1256. Dissecting Fas signaling with an altered-specificity death-domain mutant: requirement of FADD binding for apoptosis but not Jun N-terminal kinase activation.

[33] Nagata, S. (1997) Cell 88,355-365. Apoptosis by Death Factor.

[34] Perlman, R., Schiemann, W. P., Brooks, M. W., Lodish, H. F., and Weinberg, R. A. (2001) Nat. Cell Biol. 3,708-714. TGF- $\beta$-induced apoptosis is mediated by the adapter protein Daxx that facilitates JNK activation.

[35] Hollenbach, A.D., McPherson, C.J., Mientjes, E.J., Iyengar, R., and Grosveld, G. (2002) J. Cell Sci. 115,3319-3330. Daxx and histone deacetylase II associate with chromatin through an interaction with core histones and the chromatin-associated protein Dek. 


\section{FIGURE LEGENDS}

\section{Fig. 1. Physical interactions between Daxx and TSG101.}

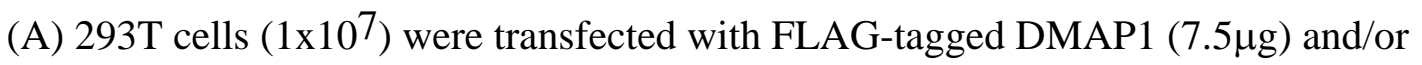

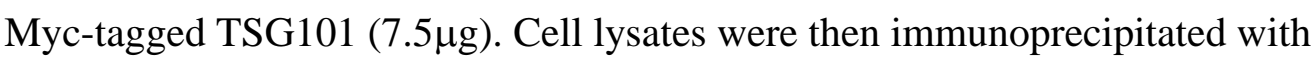
anti-FLAG antibody, and immunoblotted with anti-Myc antibody (upper panel) or anti-FLAG antibody (middle panel). Total cell lysates (1\%) were blotted with anti-Myc antibody as indicated (lower panel).

(B) $293 \mathrm{~T}$ cells $\left(1 \times 10^{7}\right)$ were transfected with FLAG-tagged Daxx $(7.5 \mu \mathrm{g})$ and/or

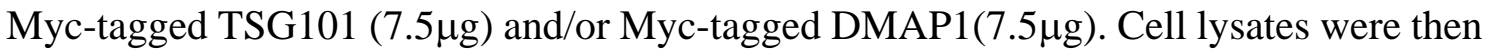
immunoprecipitated with anti-FLAG antibody, and immunoblotted with anti-Myc antibody (upper panel) or anti-FLAG antibody (middle panel). Total cell lysates (1\%) were blotted with anti-Myc antibody as indicated (lower panel).

Fig. 2. Mapping of the interacting domain between Daxx and TSG101.

(A) Schematic overview depicting interaction domains. Daxx mutant fragments used in experiments are also schematically shown. $293 \mathrm{~T}$ cells $\left(1 \times 10^{7}\right)$ were transfected with a series of Myc-tagged Daxx mutants (7.5 $\mu \mathrm{g})$ and FLAG-tagged TSG101(7.5 $\mu \mathrm{g}) .48 \mathrm{~h}$ after transfection, cells were lysed and immunoprecipitated with an anti-FLAG antibody, and immunoblotted with anti-Myc antibody (upper panel) or anti-FLAG antibody (middle panel). Total cell lysates (1\%) were blotted with anti-Myc antibody (lower panel). The asterisks indicate the migration position of Daxx deletion mutants.

(B) Domain structure of TSG101 and mutant fragments are schematically shown. 293T cells $\left(1 \times 10^{7}\right)$ were transfected with a series of Myc-tagged TSG101 mutants $(7.5 \mu \mathrm{g})$ and

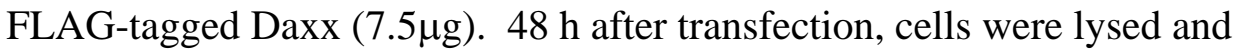


immunoprecipitated with an anti-FLAG antibody, and immunoblotted with anti-Myc antibody (upper panel) or anti-FLAG antibody (middle panel). Total cell lysates (1\%) were blotted with anti-Myc antibody (lower panel). The asterisks indicate the migration position of the full-length TSG101 or deletion mutants.

\section{Fig. 3. Colocalization of Daxx with TSG101 in the nucleus.}

(A) COS7 cells were cotransfected with FLAG-tagged Daxx and Myc-tagged TSG101 by the calcium phosphate precipitation protocol. $48 \mathrm{~h}$ after transfection, cells were fixed, reacted with an anti-Daxx polyclonal antibody and an anti-Myc monoclonal antibody, and visualized with a FITC-conjugated anti-rabbit antibody (Daxx) and a rhodamine-conjugated anti-mouse antibody (TSG101). These figures were merged. The same slide was also stained with DAPI.

(B) COS7 cells were cotransfected with FLAG-tagged Daxx and Myc-tagged TSG101CC by the calcium phosphate precipitation protocol. $48 \mathrm{~h}$ after transfection, cells were fixed, reacted with an anti-Myc polyclonal antibody and an anti-FLAG monoclonal antibody, and visualized with an FITC-conjugated anti-rabbit antibody (Daxx) and a rhodamine-conjugated anti-mouse antibody (TSG101). These figures were merged.

Fig. 4. TSG101 and Daxx cooperatively repress glucocorticoid receptor-mediated transcriptional activity.

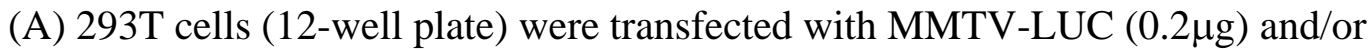
TSG101 and/or Daxx as indicated. 48 h after transfection, cells were stimulated for an additional $12 \mathrm{~h}$ for DEX $\left(10^{-7} \mathrm{M}\right)$ as indicated. LUC activities were determined. (B) 293T cells (12-well plate) were transfected with MMTV-LUC $(0.2 \mu \mathrm{g})$ and/or Daxx and/or TSG101CC as indicated. $48 \mathrm{~h}$ after transfection, cells were stimulated for an 
additional $12 \mathrm{~h}$ for DEX $\left(10^{-7} \mathrm{M}\right)$ as indicated. LUC activities were determined. The results are presented as fold induction of luciferase activity from triplicate experiments, and the error bars represent the standard deviations. 
A

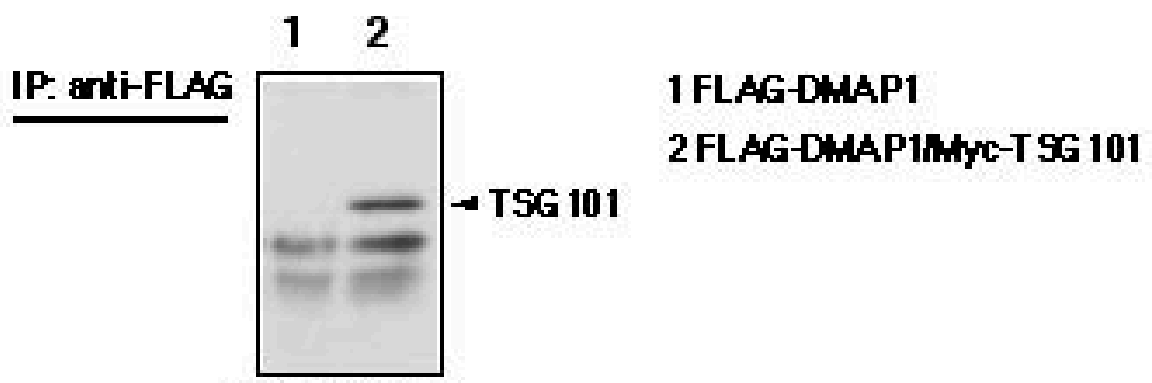

Bat: antiHhyc

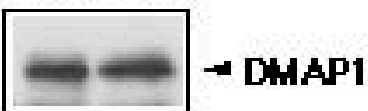

Bdot: anti-FLAG

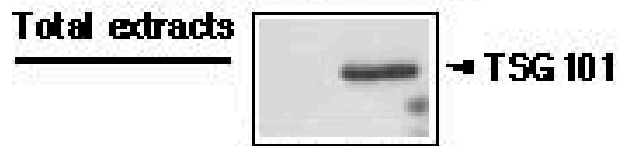

Bot: anti-Hyc

B

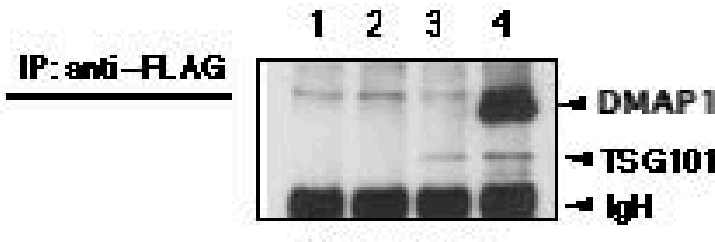

1 Mone

2 FLAGDaxx

3 FLAGDaxithe-TSG10 1

4 FLAG-Daxatyc-TSG10 1Atyc-DaAP1

BAot: anti+hyc

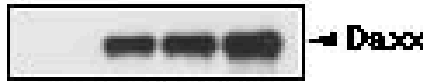

BAot: anti-PLA

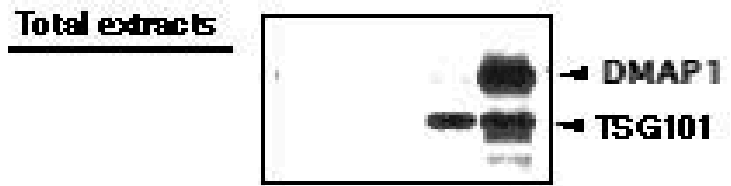

BAot: anti+hyc

Fig. 1 
A
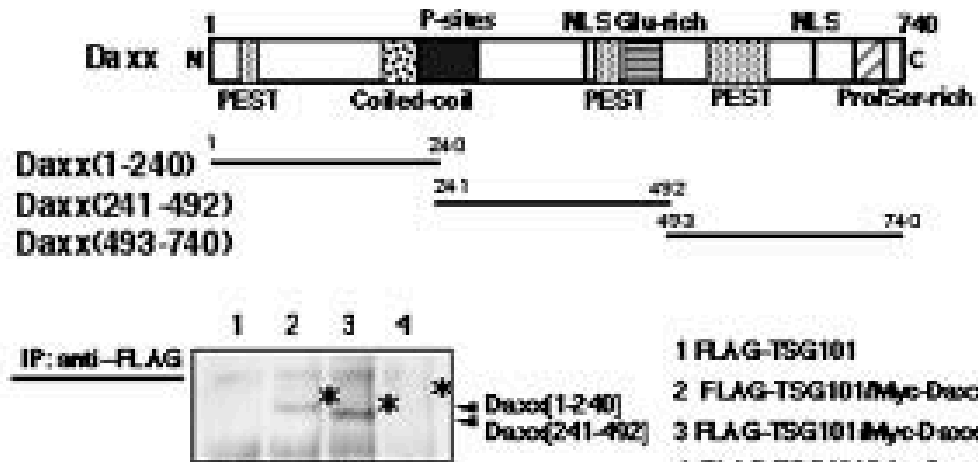

1 PLG-TSG101

2 FLAG-TSG1019ue-Daxd (1240]

3 FLG-TSG101 amycD wod(241-4he)

4 FLAG-ISGI01MAc-Dwod $493-740$ ]

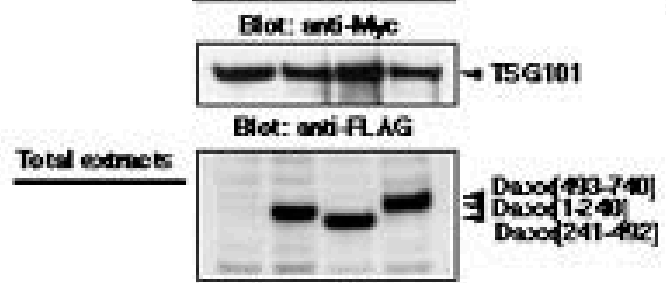

Bioc: antiane

B
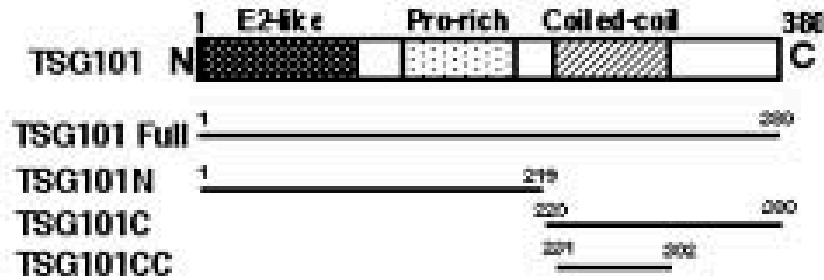
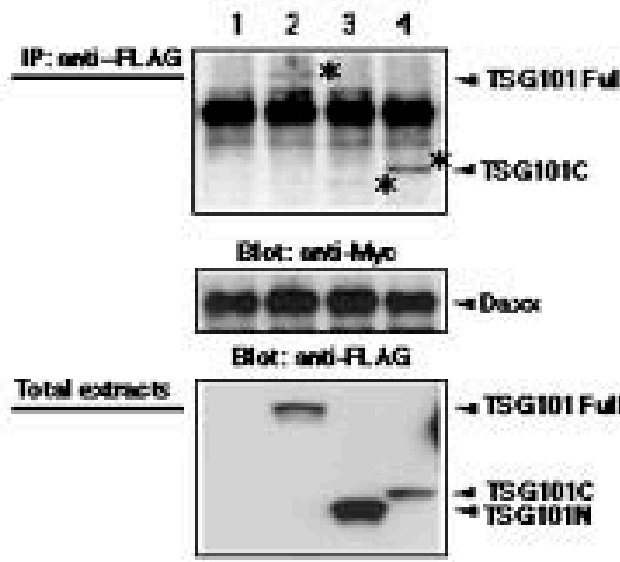

1PRGDEO

2 FLAGD wodye-TS6101Full 3 PLAG Dwodme TSG10IN 4 FLAGDacolityo-TSG101C 5 PUGDeodme-TSGIOICC 6 FLAGD wor
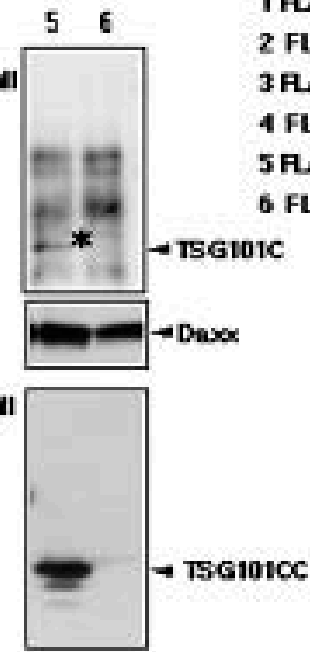

Fig. 2 
A
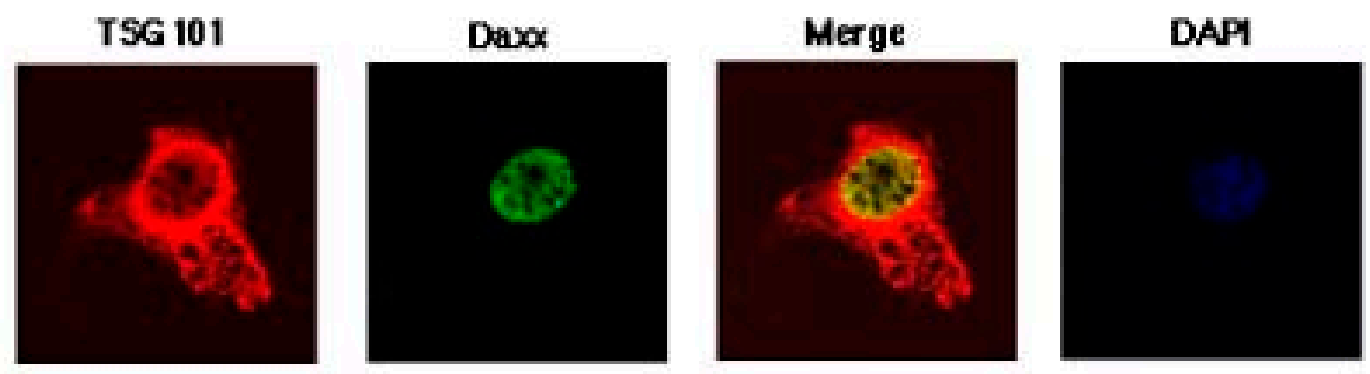

B

TSG $101 C C$
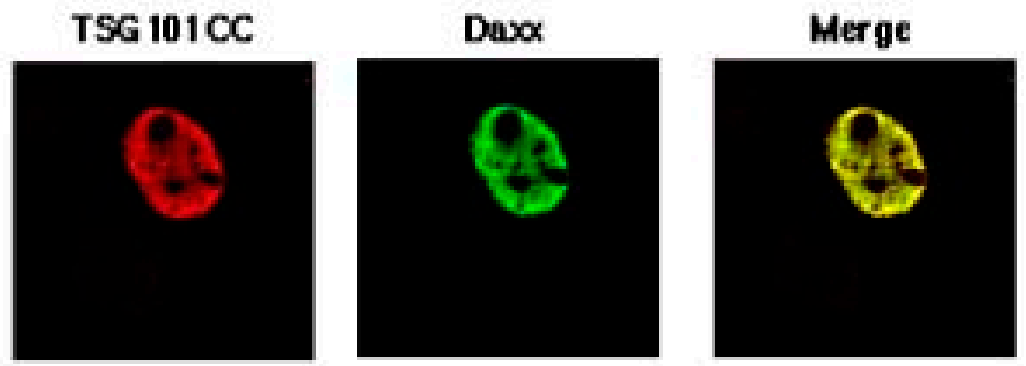

Fig. 3 


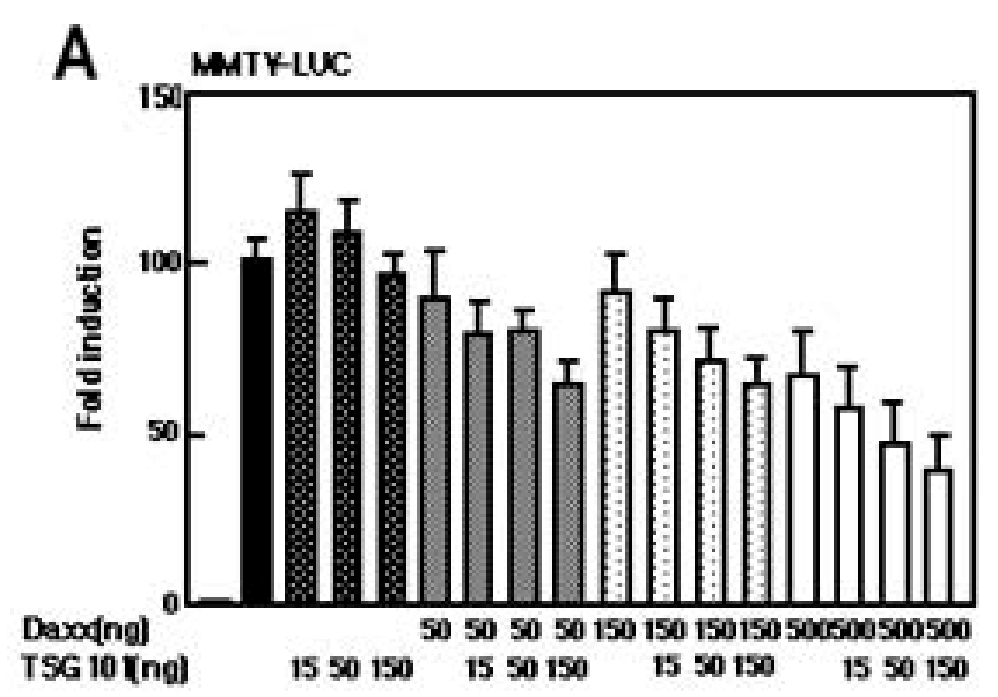

B

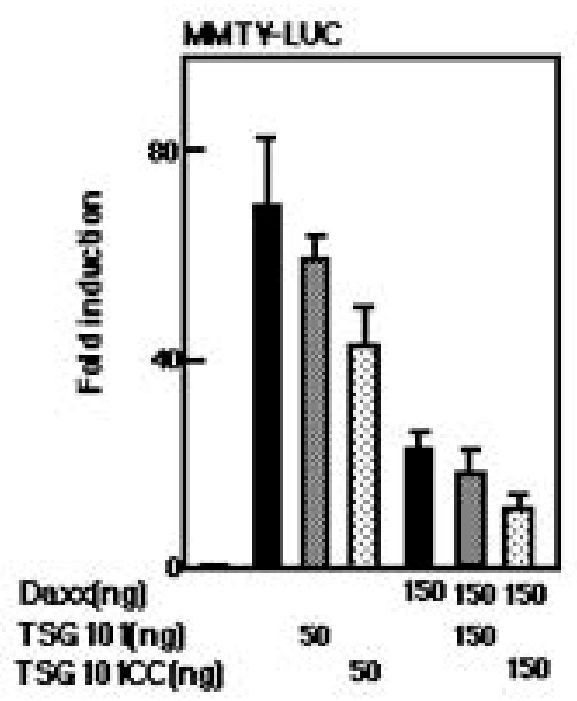

Fig. 4 